Aims/Objectives To promote the importance of HIV testing, and to see if the outreach was successful in targeting a less tested population of MSM.

Methods The promotion was determined by the "opportunities to view" key messages. Individuals tested in the bar completed a form collecting age, gender, orientation, time since last HIV test, unprotected anal (UAI) or vaginal sex. Similar information was collected from 100 patients walking into the generic service for an HIV test on the same day. Age groups $<25,26-30,31-39$, $>40$ were chosen. Results There were 890000 opportunities to view the key messages. 467 individuals tested in the gay bar, but only 441 forms were sufficiently complete for this analysis. MSM in the gay bar were younger than the clinic (54\% and 44\% respectively $<30 ; p=0.027$ ), and more likely to have never tested ( $18 \%$ and $6 \% ; p=0.007)$. In the gay bar, only $9 / 89$ (10\%) of MSM aged 31-39 had never tested, only $2 / 9$ (22\%) reporting UAI. This contrasted to the $15 \%-24 \%$ of 54 MSM in the other age groups that had never tested, $60 \%-77 \%$ of whom reported UAI. Only five MSM attending clinic had never tested, 4 (80\%) of whom were $<25$ (see abstract P7 table 1).

Discussion As well as promoting the benefits of regular testing to the wider gay community, the event was successful in reaching a less tested, but nonetheless high risk population of MSM, in particular, those $<25$.

Abstract P7 Table 1 Demographic characteristics of individuals accepting HIV testing

\begin{tabular}{lll}
\hline & Gay Bar (n=441) & Clinic (n=100) \\
\hline Male & $379(86 \%)$ & $91(91 \%)$ \\
MSM & $355(80 \%)$ & $80(80 \%)$ \\
$<25$ years & $167(38 \%)$ & $15(15 \%)$ \\
MSM $<25$ & $131(37 \%)$ & $12(12 \%)$ \\
No previous test & $101(23 \%)$ & $15(15 \%)$ \\
Never tested, reporting UAI/UVI & $52(51 \%)$ & $9(60 \%)$ \\
MSM never tested, reporting UAI & $39(62 \%)$ & $2(100 \%)$ \\
\hline
\end{tabular}

\section{P8 TESTS OF RECENT HIV INFECTION IN CLINICAL PRACTICE: THE PATIENT PERSPECTIVE}

doi:10.1136/sextrans-2012-050601c.8

${ }^{1} \mathrm{E} R$ Anderson, ${ }^{*} \mathrm{M}$ Taegtmeyer, ${ }^{3} \mathrm{~V}$ Delpech, ${ }^{4} \mathrm{~A}$ Chawla, ${ }^{3} \mathrm{~V}$ Gilbart. ${ }^{1}$ Royal Liverpool University Hospital, Liverpool, UK; 'Liverpool School of Tropical Medicine, Liverpool, UK; ${ }^{3}$ Health Protection Agency; ${ }^{4}$ Royal Liverpool Hospital, Liverpool, UK

Background A test for recent infection (avidity test) is offered for all patients newly diagnosed with HIV in England and Wales as part of an HIV incidence surveillance programme. The UK is currently the only country to return these results to individual patients.

Objectives To determine the acceptability and patient experience of receiving a RITA test result soon after HIV diagnosis.

Methods This was a qualitative study using semi-structured interviews. 14 people recently diagnosed with HIV who had a RITA result consented to participate. Analyses were based on the framework method using N-Vivo software. Interviews were transcribed, coded and emergent themes identified.

Results All participants agreed that the more information available to them about the possible duration of infection the better. Unsurprisingly the HIV diagnosis and the emotions and practical issues associated with it had far more impact than the RITA result. None of the participants experienced any problems with former partners as a consequence of their RITA result although some could see the theoretical potential for such problems. "Recently infected" RITA results were felt to be potentially useful for identifying "at risk" partners. However partner notification was not altered in the study group because the individuals concerned had other reasons to suspect recent infection. Other major themes identified were the perceived stigma; the difficulty of sharing the diagnosis of HIV with family and friends; and the many conflicting emotions that people had to deal with at diagnosis including anger, grief, self-blame, fear and depression.

Conclusion RITA testing is a potentially useful epidemiological tool. These interviews demonstrated that receiving a RITA result, while useful to some people, is a minor issue compared with dealing with the HIV result itself. Reassuringly none of the participants reported negative outcomes from receiving the RITA result.

\section{P9 $\quad$ SUSTAINABLE AND EMBEDDED OPT OUT HIV TESTING ON AN ACUTE MEDICAL ADMISSIONS UNIT}

doi:10.1136/sextrans-2012-050601c.9

A Palfreeman, ${ }^{*}$ G Mckinnon. University Hospitals Leicester, Leicester, UK

Background Opt out testing for HIV in our hospital's acute medical unit (AMU) had been successfully piloted between August 2009 and September 2010. Our trust was selected a pilot site to implement the 2008 national HIV testing guidelines as an area of high HIV prevalence outside London. (Data from this pilot were presented at BASHH in Gateshead in 2010). However, could opt out testing for HIV on an AMU be sustained beyond the pilot?

Methods HIV testing in the pilot was embedded into the normal working of the AMU, clinical aides did the phlebotomy, medical admission proformas were modified to include HIV testing and consent was obtained by a widely distributed information leaflet. One of the GU consultants attended the AMU to remind doctors to test several times a week. After the pilot finished, the GU consultant stopped attending the ward HIV testing is now part of routine care on the AMU. It is explained at each junior doctors induction which includes a patient video. Electronic blood test requests for AMU automatically include an HIV test as part of the AMU blood bundle set. A CQUIN target that $25 \%$ of all admissions are to be tested for HIV has been set by commissioners. Testing has been expanded from the 16-60 age range to the 16-80 age range.

Results The rate of HIV testing has risen from 80 a month to $140 \mathrm{a}$ month in 2011 the number of new + ves diagnosed has risen from 10 per year to 25 in 2011

Conclusion Sustainable opt out testing for HIV on an AMU can be achieved by embedding the testing process in the clinical pathways that already exist upon an AMU. This requires no extra manpower or resources to achieve within an AMU setting. Since the withdrawal of consultant support our average testing rate has gone up. Our model for opt out HIV testing is therefore sustainable, requires little extra resource and should be easy to reproduce in other centres.

\section{P10 VITAMIN D LEVELS IN A SAMPLE OF HIV+ PATIENTS}

doi:10.1136/sextrans-2012-050601c.10

G Brook, ${ }^{*}$ J Alagaratnam, K Barai. North West London Hospitals NHS Trust, Central Middlesex Hospital, London, UK

Background In the last few years it has been highlighted that vitamin $\mathrm{D}$ deficiency is common in the general population however, the extent of the problem in different HIV+ cohorts is less clear. Aims In a cohort of HIV+ patients in North West London we looked at the vitamin D levels to see what the prevalence of deficiency was and see if there were any correlates with ethnicity or season.

Methods All HIV+ patients at this centre and who had a recorded vitamin $\mathrm{D}$ test result in the last 2 years were identified. The first result while not on treatment was recorded. The patients' electronic 
case records were examined to identify any correlates with the results. Vitamin D was classified as deficient, insufficient or adequate with total 25 -hydroxy vitamin $\mathrm{D}$ blood levels of $<12.5$, 12.5-50 and $>50 \mathrm{nmols} / \mathrm{l}$ respectively.

Results 143 patients had a least one vitamin D level measured. Of these 7 (5\%) had vitamin D deficiency, 83 (58\%) insufficient levels and $53(37 \%)$ had adequate levels. Comparing patients who had levels tested in the winter months with those tested in the summer, they were more likely to show deficient or insufficient levels than adequate levels $(44 / 90(49 \%)$ vs $6 / 53(11 \%)$ p < 0.0001). All patients who showed gross deficiency were black or Asian but there wasn't a correlation between ethnicity and insufficient/deficient levels. Three (42\%) of the patients with deficient levels had a raised serum alkaline phosphotase and/or a low serum phosphate.

Conclusions High rates of vitamin D deficiency and insufficiency were found in this cohort, only part of which is explained by doing the test in the winter months. Some evidence of metabolic effects of deficiency on bone was found

\section{P11 HIV TEST UPTAKE IN THE GU CLINIC: JUNIORS HOT ON THE HEELS OF THE CONSULTANTS}

doi:10.1136/sextrans-2012-050601c.11

0 Isabel, ${ }^{*}$ M D Portman, R Giuntini, CMW Stewart, J D Wilson. Leeds General Infirmary

Background Data from the Health Protection Agency shows that $25 \%$ of people living with HIV in the UK are unaware of their diagnosis. This has serious health implications for them and others.

The National Strategy for Sexual Health and HIV set a target of $60 \%$ testing uptake by $2007.69 \%$ of STI attendees at GUM clinics in England in 2010 were tested for HIV. Regional data from the Health Protection Unit shows a 70\% uptake of HIV testing in women attending our clinic. Recent data from the UK and Australia has documented variable HIV testing rates among clinicians with a trend towards more junior doctors being more likely to offer a test. Objectives To identify HIV testing rates according to grade of clinician.

Methods Women attending our service as new or rebook patients between March 2009 and January 2010 were seen by clinicians representing eight different grades of staff. The women's response to the offer of an HIV test was recorded. These data were analysed according to the grade of the staff member.

Results 3973 women were seen by 42 different clinicians. 2982 accepted an HIV test (P1A); 980 declined a test (P1B) and 11 deferred testing (P1C) (see abstract P11 table 1).

Conclusions There are many perceived barriers to HIV testing and it is often quoted that testing rates are lowest among non-specialists.
However, there is little published data on the impact of the individual clinician on HIV testing. Encouragingly, our data has shown that more junior staff, having trained in an environment where HIV is increasingly recognised as a treatable condition, are confident to offer HIV testing and do so as effectively as consultants. We should learn from them with respect to promoting HIV testing in our clinics and not underestimate the value of ongoing training and support in this important area.

\section{P12 HIV TESTING: ARE WE DOING ENOUGH? A STAFF AWARENESS SURVEY IN EAST MIDLANDS}

doi:10.1136/sextrans-2012-050601c.12

J Dhar, ${ }^{*}$ F Nyatsanza. University Hospitals of Leicester, Leicester, UK

Background The overall HIV prevalence in East Midland is low and since 2000 most of the new cases in our region continue to be diagnosed in the same three Primary Care Trusts, peaking in the 35-39-year-old adults in 2009/2010. HIV uptake rates vary from $69 \%$ to $80 \%$ and high coverage remains a possibility as demonstrated by $96 \%$ antenatal uptake rates.

Objective To collect information from hospital and community based staff about HIV testing, to develop effective regional strategies.

Methods A 10 min online Questionnaire was completed by staff members via Survey Monkey this included gender, age, time since qualification, experience of HIV test use, training issues etc.

Results 1067 responses have been analysed, $>75 \%$ of respondents being female, representing a wide range of specialties including general practice. $21 \%, 28 \%, 28 \%, 22 \%$ are aged between $20-30$ years, $30-40$ years, $40-50$ years and $>50$ years respectively. $25.5 \%, 21 \%, 25 \%, 29 \%$ of the respondents were qualified $<5$ years, 5-10 years, $10-20$ years, more than 20 years respectively. $46.8 \%$ had no prior experience of offering an HIV test. $39.9 \%$ felt they had the necessary skills a similar proportion $39.5 \%$ felt they lacked them. $21.8 \%$ of the respondents would have concerns offering a test. While the majority $81.6 \%$ agreed with NICE guidance on expanded testing $57.7 \%$ would require further training, $50.2 \%$ saying they would be unable to answer patients questions on HIV testing. 85.1\% agreed that HIV updates need to be incorporated in induction provided to health care professionals with $45.9 \%$ stating that current methods were not adequate.

Conclusions The survey highlights that while health care professionals are aware of the need to have a HIV test nearly half of them have never offered one and a large proportion have identified anxieties around consultation. Inadequate training was cited as a major factor for not considering or offering a test.

Abstract P11 Table 1 HIV testing rates by different health care professionals

\begin{tabular}{|c|c|c|c|c|c|c|c|c|c|}
\hline & Band 5 nurses & Band 6 nurses & FY1 & ST2 & GP & SAS & Reg & Cons & Total \\
\hline No of staff & 6 & 7 & 6 & 4 & 2 & 2 & 9 & 6 & 42 \\
\hline No of consultations & 169 & 442 & 140 & 207 & 252 & 837 & 996 & 930 & 3973 \\
\hline P1A & 122 & 325 & 112 & 171 & 196 & 572 & 753 & 731 & 2982 \\
\hline P1B & 47 & 115 & 28 & 36 & 52 & 265 & 239 & 198 & 980 \\
\hline P1C & 0 & 2 & 0 & 0 & 4 & 0 & 4 & 1 & 11 \\
\hline $\begin{array}{l}\% \text { Patients who } \\
\text { decline HIV test }\end{array}$ & 27.8 & 26.0 & 20.0 & 17.4 & 20.6 & 30.6 & 24.0 & 21.3 & 24.7 \\
\hline $\begin{array}{l}\text { OR }(95 \% \mathrm{CI}) \text { patient } \\
\text { having an HIV test } \\
\text { compared to } \\
\text { other grades }\end{array}$ & $\begin{array}{l}1.18 \\
(0.84 \text { to } 1.67)\end{array}$ & $\begin{array}{l}1.08 \\
(0.86 \text { to } 1.36)\end{array}$ & $\begin{array}{l}0.76 \\
(0.49 \text { to } 1.15)\end{array}$ & $\begin{array}{l}0.63 \\
(0.44 \text { to } 0.19)\end{array}$ & $\begin{array}{l}0.78 \\
(0.57 \text { to } 1.07)\end{array}$ & $\begin{array}{l}1.57 \\
(1.33 \text { to } 1.86)\end{array}$ & $\begin{array}{l}0.95 \\
(0.81 \text { to } 1.13)\end{array}$ & $\begin{array}{l}0.78 \\
(0.66 \text { to } 0.93)\end{array}$ & \\
\hline p Value & 0.38 & 0.52 & 0.23 & 0.016 & 0.14 & $<0.0001$ & 0.60 & 0.007 & \\
\hline
\end{tabular}

